\title{
An Assumption of Wearing Calculation with the Miner Fatigue Accumulate Theory
}

\author{
Liu Huran ${ }^{*}$
}

Department of Mechanical Engineering, Zhejiang University of Science and Technology, Hang zhou, Zhejiang, China

\begin{abstract}
This paper finds the similarity between the fatigue strength calculation and the IBM wearing calculation method. The Miner Fatigue Accumulate Theory of the unsteady loading has been applied into the calculation of the wearing of the unsteady loading, and further more, has been applied into the case when the load variations are continuous. Aims and objectives: We can make use of the Miner Fatigue Accumulate Theory both for the unsteady fatigue wear as well as unsteady fatigue load.
\end{abstract}

\section{THE SIMILARITY BETWEEN THE FATIGUE STRENGTH CALCULATION AND THE IBM WEAR- ING CALCULATION METHOD}

The background subject area is the Miner Fatigue Accumulate Theory and the IBM method. The novelty of the paper is to make use of the Miner Fatigue Accumulate Theory for the unsteady fatigue wear.

IBM (International Business Machine Cooperation Lit.) is the world's most furious producer of computer. During the research for the friction and wearing of the hard disk of the computer, the IBM engineers developed a method to calculate the wearing of material. This method is called the IBM wearing calculation method. According to IBM wearing calculation method, the relationship between the number of "pass" to insure the "Zero Wear" and the maximum shearing stress has the following relationship [1-3]:

$\tau_{\max }^{9} \gamma=\mathrm{const}=2000 \gamma_{\mathrm{R}} \tau_{y}^{9}$

In the equation: $\gamma$ : The number of "pass"

$\gamma_{R}$ : The parameter of wearing

$\tau_{y}:$ The limit of shear stress

It is not difficult to find out the similarity between the IBM wearing calculation method and the fatigue strength curve.

$\sigma^{m} N=\sigma_{r}^{m} N_{0}$

Here, the number of "pass" $\gamma$ corresponds to the cycle of fatigue N. $\tau_{\max }$ corresponds to the stress $\sigma$. The limit of fatigue $\sigma_{r}$ corresponds to the $\gamma_{R} \tau_{y}$, cardinal number of cycle $N_{0}$ corresponds to 2000 .

*Address correspondence to this author at the Department of Mechanical Engineering, Zhejiang University of Science and Technology, Hang zhou, Zhejiang, China; E-mail: 1hrcsu@163.com

\section{THE MINER FATIGUE ACCUMULATE THEORY FOR IBM WEARING CALCULATION}

For the unsteady fatigue load, suppose that, under the load $\sigma_{1}, \sigma_{2}, \ldots . \sigma_{m}$, the cycles of fatigue are $n_{1}, n_{2}, \ldots . . n_{m}$, respectively. For every cycle of fatigue, the damages to the material are $\frac{1}{N_{1}}, \frac{1}{N_{2}}, \ldots . \frac{1}{N_{m}}$, respectively. The damages caused by $\sigma_{1}, \sigma_{2}, \ldots \sigma_{m}$ will be $\frac{n_{1}}{N_{1}}, \frac{n_{2}}{N_{2}}, \ldots \frac{n_{m}}{N_{m}}$, respectively. According to Miner Fatigue Accumulate Theory [4], the material will fail when and only when:

$\frac{n_{1}}{N_{1}}+\frac{n_{2}}{N_{2}}+\ldots .+\frac{n_{m}}{N_{m}}=\Sigma \frac{n_{i}}{N_{i}}=1$

For the unsteady fatigue wear, we also suppose that, under the load $\tau_{\max 1}, \tau_{\max 2}, \ldots . \tau_{\max m}$, the cycles of wear are $n_{1}, n_{2}, \ldots . n_{m}$, respectively. For every cycle of fatigue, the damages to the material are $\frac{1}{\gamma_{1}}, \frac{1}{\gamma_{2}}, \ldots . \frac{1}{\gamma_{m}}$, respectively. The damages caused by $\tau_{\max 1}, \tau_{\max 2}, \ldots . \tau_{\max m}$ will be $\frac{n_{1}}{\gamma_{1}}, \frac{n_{2}}{\gamma_{2}}, \ldots . \frac{n_{m}}{\gamma_{m}}$ respectively. Similar to Miner Fatigue Accumulate Theory, it is supposed that the material will fail when and only when (Fig. 1):

$\frac{n_{1}}{\gamma_{1}}+\frac{n_{2}}{\gamma_{2}}+\ldots .+\frac{n_{m}}{\gamma_{m}}=\Sigma \frac{n_{i}}{\gamma_{i}}=1$

\section{THE INTEGRAL FORM OF MINER FATIGUE AC- CUMULATE THEORY}

Because of that:

$$
\gamma_{1}=2000\left(\frac{\tau_{y} \gamma_{R}}{\tau_{\max 1}}\right)^{9}, \gamma_{2}=2000\left(\frac{\tau_{y} \gamma_{R}}{\tau_{\max 2}}\right)^{9}, \gamma_{i}=2000\left(\frac{\tau_{y} \gamma_{R}}{\tau_{\max i}}\right)^{9}
$$

We have: 
$\Sigma \frac{n_{i}}{\gamma_{i}}=\Sigma \frac{n_{i}}{2000}\left(\frac{\tau_{\max i}}{\tau_{y} \gamma_{R}}\right)^{9}=\frac{1}{2000\left(\tau_{y} \gamma_{R}\right)} \Sigma \tau_{\max i}^{9} n_{i}$

If for every shear stress, the corresponding number of "pass" is rather small, and the shear stress and the corresponding number of "pass" varied continuously, then, the non continuous Miner Fatigue Accumulate Theory becomes continuous Miner Fatigue Accumulate Theory. The integral form of the Miner Fatigue Accumulate Theory is [5]:

$\int_{0}^{\gamma}\left[\tau_{\max }(n)\right]^{9} d n=2000\left(\gamma_{R} \tau_{y}\right)^{9}$

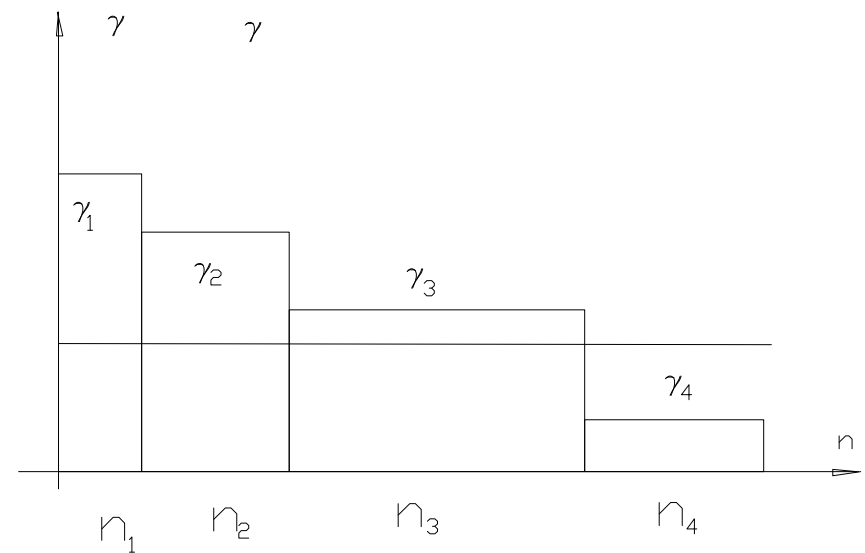

Fig. (1). The Miner fatigue accumulate theory for IBM wearing calculation.

\section{CONCLUSIONS}

This paper finds the similarity between the fatigue strength calculation and the IBM wearing calculation method. The Miner Fatigue Accumulate Theory of the unsteady loading has been applied to the calculation of the wearing of the unsteady loading, and further more, has been applied to the case when the load variations are continuous.

\section{REFERENCES}

[1] Yang ZM. The Basis of the Tribology Design. Industrial Publisher: Beijing, China 1990

[2] Jones MH, Scott D. Industrial Tribology. Elsevier: Amsterdam 1983.

[3] Cameron A. Basic Lubrication Theory. $3^{\text {rd }}$ ed. Ellis Horwood Limited: New York 1981.

[4] Joseph ES. Mechanical Engineering Design. $3^{\text {rd }}$ ed. McGraw-Hill: New York 1977.

[5] Huran L. Revised adhesive theory with pollute film on metal surfaces. Zhejiang University of Science and Technology: Hangzhou, China 2008.

(C) Liu Huran; Licensee Bentham Open.

This is an open access article licensed under the terms of the Creative Commons Attribution Non-Commercial License (http://creativecommons.org/licenses/ by$\mathrm{nc} / 3.0 /$ ) which permits unrestricted, non-commercial use, distribution and reproduction in any medium, provided the work is properly cited. 